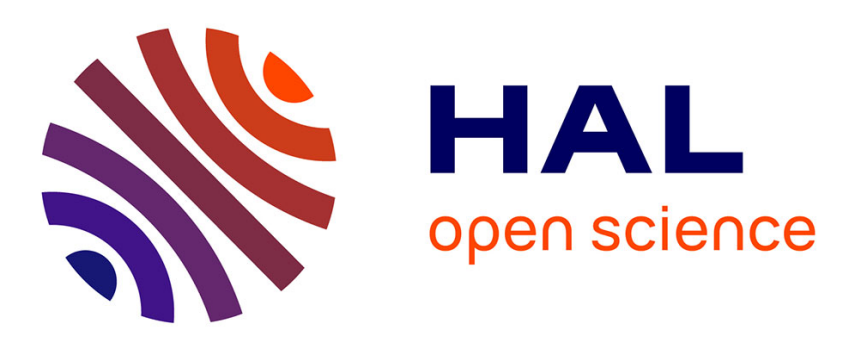

\title{
Stability of a Liquid Jet Impinging on Confined Saturated Sand
}

Jérémy Vessaire, Germán Varas, Sylvain Joubaud, Romain Volk, Mickaël Bourgoin, Valérie Vidal

\section{- To cite this version:}

Jérémy Vessaire, Germán Varas, Sylvain Joubaud, Romain Volk, Mickaël Bourgoin, et al.. Stability of a Liquid Jet Impinging on Confined Saturated Sand. Physical Review Letters, 2020, 124 (22), pp.224502. 10.1103/PhysRevLett.124.224502 . hal-02929125

\section{HAL Id: hal-02929125 \\ https://hal.science/hal-02929125}

Submitted on 12 Oct 2020

HAL is a multi-disciplinary open access archive for the deposit and dissemination of scientific research documents, whether they are published or not. The documents may come from teaching and research institutions in France or abroad, or from public or private research centers.
L'archive ouverte pluridisciplinaire HAL, est destinée au dépôt et à la diffusion de documents scientifiques de niveau recherche, publiés ou non, émanant des établissements d'enseignement et de recherche français ou étrangers, des laboratoires publics ou privés. 


\title{
Stability of a liquid jet impinging a confined saturated sand
}

\author{
Jérémy Vessaire ${ }^{1}$, Germán Varas ${ }^{2}$, Sylvain Joubaud ${ }^{1,3}$, Romain Volk ${ }^{1}$, \\ Mickaël Bourgoin ${ }^{1}$ and Valérie Vidal $^{1}$ \\ 1 Univ Lyon, ENS de Lyon, Univ Lyon 1, CNRS, Laboratoire de Physique, F-69342 Lyon, France \\ ${ }^{2}$ Instituto de Física, Pontificia Universidad Católica de Valparaíso, Av. Universidad 330, Valparaíso, Chile and \\ ${ }^{3}$ Institut Universitaire de France (IUF)
}

(Dated: April 10, 2020)

\begin{abstract}
This work focuses on the dynamics of a liquid jet impacting the surface of a confined, immersed granular bed. Although previous works have considered the erosion process and surface morphology, less attention has been given to the jet hydrodynamics. Based on laboratory experiments, we show that when the liquid jet forms a crater, two situations arise. In the case of weak/no erosion or open craters, the jet is stationary. For vertical or overhanging crater walls, the jet displays a wide range of behaviors, from quasi-periodic oscillations to symmetry breaking and exploration of different states in time. An analysis of the different system states leads to the emergence of a bifurcation diagram depending on a dimensionless parameter, $J$, comparing the jet impact force to the force necessary to eject a grain. The frequency of the jet oscillations depends on the inertial velocity, the jet dispersion and the ratio between the injector cross-section and the confinement length.
\end{abstract}

Crater formation by impacting a granular material is ubiquitous in nature, from raindrops falling on sandy deserts to meteorites impacting moons and planets. Due to the complexity of the underlying physical mechanisms, it has raised the curiosity of scientists since more than a century (see $[1,2]$ and references therein). In the last decades, however, the human race to space and deep oceans has brought forward new challenges related to the formation of craters by impinging jets. On the one hand, since the Apollo and Viking programs, erosion and cratering mechanisms have focused great interests as they have direct consequences on hazards for retrograde rockets landing on planetary regoliths - reduced visibility, vehicle damage or uneven landing surface [37]. On the other hand, recent technical developments for either space exploration or deep-sea mining allow mineral extraction by impacting jets [8-11], such as the Touchand-Go Sample Acquisition Mechanism (TAGSAM) for asteroid sampling [12]. Understanding and quantifying the mechanisms at stake is not only a challenge for hazard mitigation but also, for deep-sea mining, a crucial need to assess potential environmental effects of largescale sediments resuspension $[8,9,13,14]$. Since the pioneering work of Aderibigbe et al. [15], many studies of two-phase systems with gas or liquid jets eroding a dry or immersed granular bed have proposed an accurate description of the erosion threshold, bedload transport or crater morphology [6, 16-22]. However, less attention has been given to the possible appearance of flow instabilities, which may lead to drastic consequences for lunar, planetary or asteroid landing hazards. Metzger et al. pointed out crater depth oscillations, which seem to "correlate to periodic avalanching of the outer crater" [4]. Later on, Clark and Behringer reported a horizontal symmetry breaking when "the sidewalls of the inner crater range from nearly vertical to overhanging and a circular-like flow pattern emerges", leading to strongly asymmetric craters [6]. However, to our knowledge, none of these studies focused on the dynamics of the jet itself. Yet it is well-known, since the first observation of the edge-tone phenomenon by Sondhaus in 1854 [23], that jets impacting on a solid obstacle or confined in a cavity can develop self-sustained oscillations [24-32]. Do these oscillations arise when the jet impacts a porous medium? What is the coupling with crater morphology? The present work focuses on the stability of a liquid jet when impacting a confined saturated granular bed. We quantify both the jet hydrodynamics and the soil surface morphology, and demonstrate the appearance of a new instability, based on the fluid-particles coupling.

The experimental device consists of a Hele-Shaw cell (40 cm width, $15 \mathrm{~cm}$ height, gap $e=2.2 \mathrm{~mm}$ ) partially filled with glass beads (Sovitec glass spheres) of diameter $d=750 \mu \mathrm{m}$, immersed in water. The particles have a density $\rho_{p}=2300 \mathrm{~kg} \cdot \mathrm{m}^{-3}$ and a density difference $\Delta \rho=\rho_{p}-\rho_{f}$ with the surrounding fluid, with $\rho_{f}=1000 \mathrm{~kg} \cdot \mathrm{m}^{-3}$. In all experiments, the granular layer height is fixed to $5 \mathrm{~cm}$ so that the maximum of the scour is always far from cell bottom. Between each experiments, the grains are stirred gently and then leveled with a ruler to obtain a horizontal free surface. This protocol ensures reproducible results. Four different impingement heights $h=[1,3,5,8] \mathrm{cm}$ are used. A home-made push-syringe generates a water jet at constant flow-rate $Q$ through a cylindrical injector of inner diameter $1.4 \mathrm{~mm}$ (cross-sectional area $S$ ) located at a height $h$ above the granular bed. The water level remains constant and wellabove the injector throughout the entire experiment using an exhaust hole located at the top of the cell. To perform Particle Image Velocimetry (PIV) analysis, the liquid is seeded with isodense tracers (Cospheric, diameter $212 \mu \mathrm{m})$. Comparison with experiments without tracers show that they do not affect the erosion process and crater morphology. Images are recorded with 


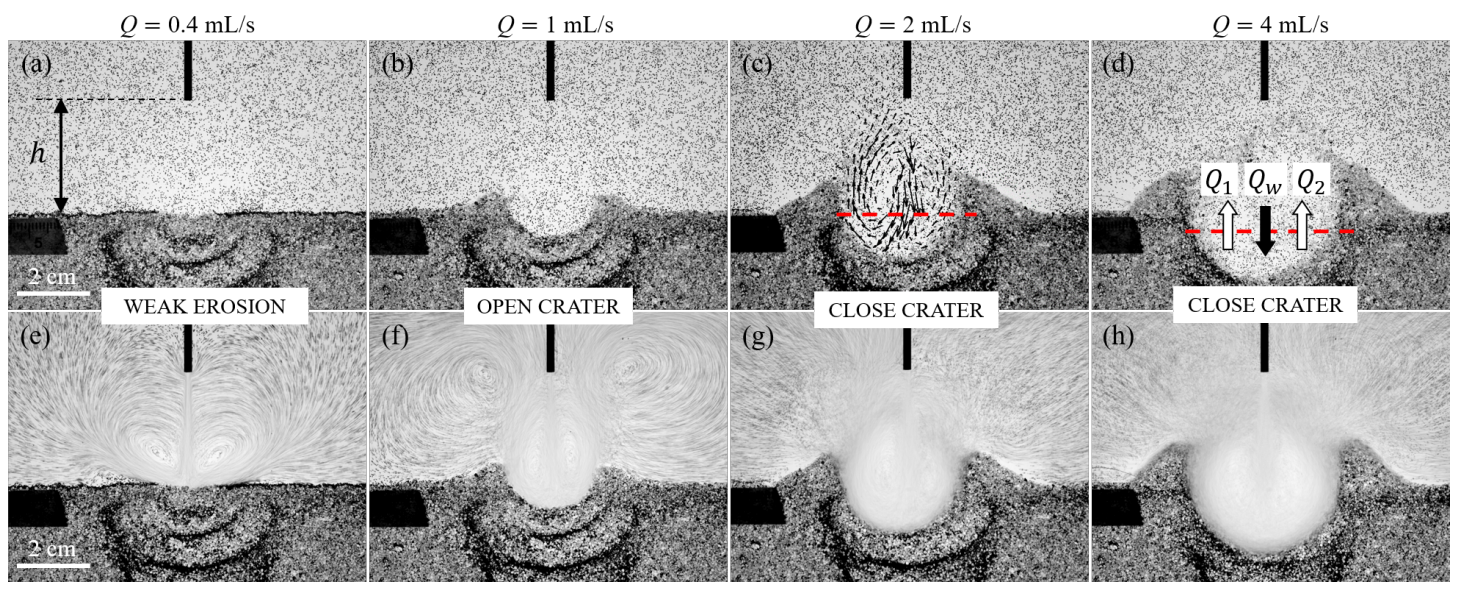

Figure 1. Crater morphology for different liquid flow rates $Q[h=3 \mathrm{~cm}]$. The upper panel (a-d) shows a snapshot and the lower panel (e-h) displays the average over the last 1000 images. (c) shows an example of PIV computation. The red dashed lines in (c,d) display the section upon which the incident (downward) flux $Q_{w}$ and the upward side fluxes $Q_{1}$ and $Q_{2}$ are computed.

a fast camera (Phantom Miro M110) at 1600 fps (size $\left.768 \times 1024 \mathrm{pix}^{2}\right)$, and the PIV field is computed by means of the open source code PIVLab [33, 34]. The contrast between the tracers and the background and glass beads is increased by means of a contrast limited adaptive histogram equalization (CLAHE) algorithm for each image. To get the largest velocity range from the nozzle to the crater bottom, a multipass PIV method with an interrogation window from $128 \times 128$ to $16 \times 16$ pixels is applied. Finally, to check the reproducibility and increase the statistics, each experiment with fixed parameters $(h, Q)$ is repeated 5 times.

At low flow-rate $Q$ and large $h$, the liquid jet is not strong enough to form a crater (Fig. 1a). As the jet speed increases or the impingement height decreases, a crater is formed (Fig. 1b,c,d). After a rapid transient, the crater morphology reaches a state in which it only evolves slowly in time (see Supplemental Material A.1 and Fig.S1). Figure 2 displays the crater morphology in this latter regime, in the parameters space $(h, Q)$. We recover the classical morphologies described in the literature [15]: weak $(\square)$ or no erosion $(\times)$, strongly deflected jet regimes, which generate a peculiar crater morphology resembling an indentation ( + , see Supplemental Material A.2), or well-developped craters (central and bottom pictures, inset Fig. 2). The open craters $(\nabla)$ are distinguished from the close craters $(\bigcirc)$ by analysing both the movies and the average intensity over the last $1000 \mathrm{im}-$ ages. Craters are defined as close when the angle between the horizontal and at least one of their inner walls is equal or larger than $90^{\circ}$ (vertical or overhanging wall, see Supplemental Material A.3). This criterion matches with the observation for open craters of buckling streamlines on each side of the impacting jet, due to the return flow which is not trapped inside the crater (Fig. 1e,f).

Coupling PIV analysis of the fluid flow and crater mor- phology evidences a region where the impinging jet does not have the classical self-similar profile and displays a non-stationary behavior (Fig. 2, gray region). We denote $Q_{w}$ the incident flux and $Q_{i}(i=1,2)$ the reflected fluxes on each side of the incident jet, computed as the spatial average of the downward and upward liquid flows through the horizontal line at the center of the crater (Fig. 1d).

Figures 3a-d display the time evolution of $Q_{1}$ and $Q_{2}$ for different jet dynamics. Granular layers with no erosion or open craters are characterized by a stationary jet, with symmetric fluxes (Fig. 3a). Jets in close craters, on the contrary, exhibit a wide range of dynamics, from fully asymmetric (Fig. 3b) to symmetric with self-sustained oscillations (Fig. 3d), including the exploration of different

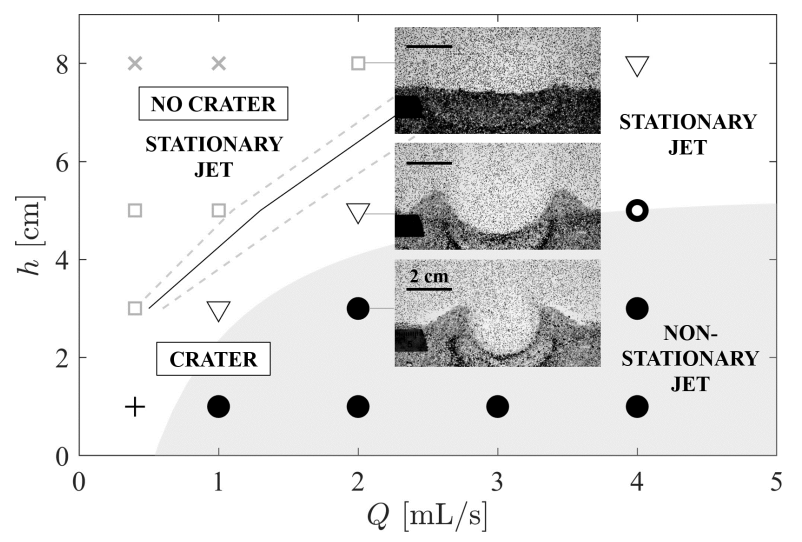

Figure 2. Crater morphology and jet stability in the phase space $(Q, h) . \times$ no erosion; $\square$ weak erosion; + jet indentation; $\nabla$ open crater; $\bigcirc$ close crater with stationary (black \& white) or non-stationary (black) jet. Solid black line: $J=0.6$ (equation 1) for the jet angle determined experimentally, with error estimation (dashed gray lines). The gray zone (guide for the eye) shows the existence of jets oscillations. 

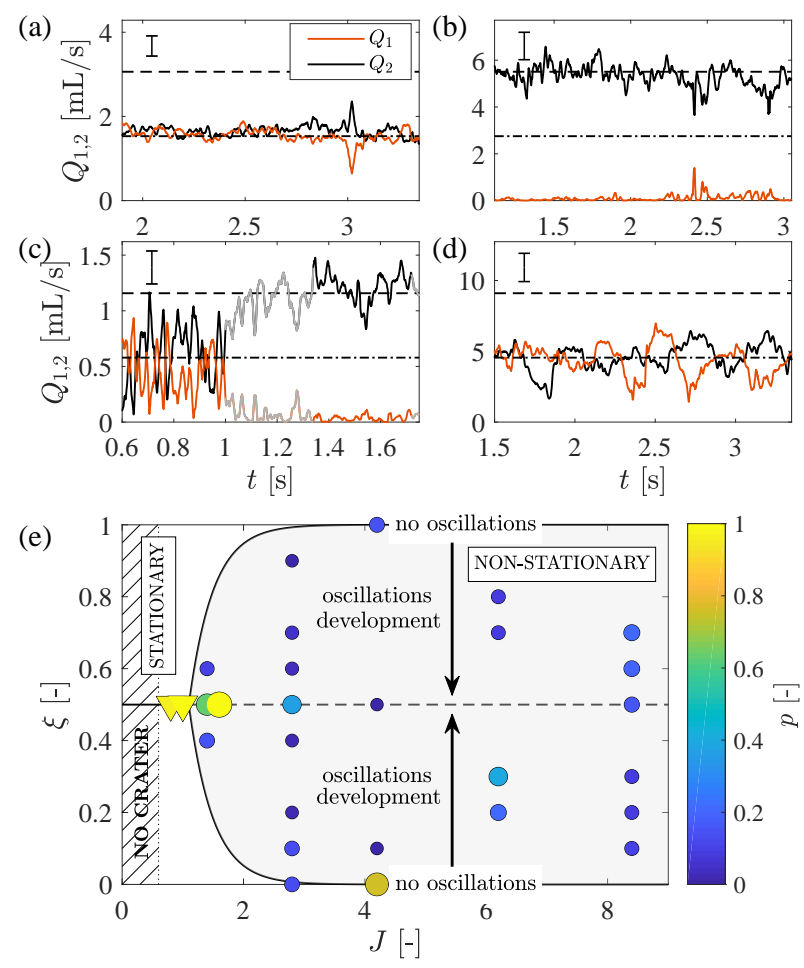

Figure 3. (a-d) Different flow regimes $\left[Q_{1}\right.$, orange; $Q_{2}$, black; $\left\langle Q_{w}\right\rangle$, dashed where $\langle$.$\rangle denotes the time average (errorbar$ $=$ standard deviation of $\left\langle Q_{w}\right\rangle$ fluctuations); $\left\langle Q_{w} / 2\right\rangle$, dashdotted] $(h[\mathrm{~cm}], Q[\mathrm{~mL} / \mathrm{s}], J)$. (a) Symmetric $(3,1,0.95)$; (b) asymmetric $(1,2,4.18)$; (c) different states $(1,1,2.86)$, the gray parts of the signals indicate the transition regions; (d) quasi-periodic oscillations $(3,4,2.78)$. (e) System asymmetry $\xi$ as a function of $J$. Hatched region: no crater formation, $\nabla=$ open craters, $\bigcirc=$ close craters. Gray zone: nonstationary jet which explores different states. The colorscale gives the probability $p$ for the system to be in a state $\{\xi\}$ for a given $J$.

states in time (Fig. 3c). A system state is defined as a constant mean value of $Q_{1,2}$, superimposed with fluctuations. Note that except for fully asymmetric states, jets in close craters always exhibit fluctuations or quasiperiodic oscillations of $Q_{1}$ and $Q_{2}$ in phase opposition, corresponding to lateral displacements of the incident jet. The typical time of these oscillations or states exploration is of the order of 0.1 to $1 \mathrm{~s}$, during which the global crater morphology does not exhibit any significant change (see Supplemental Material, Fig.S1).

To characterize the system dynamics, we introduce a dimensionless parameter adapted from the erosion parameter $E$ previously defined for submerged impinging circular jets as the ratio between the force exerted by the impacting jet on a bed particle located under the jet at the original bed surface to its resistive force [15]. It is adapted here to the confined geometry and scales as the velocity ratio of the jet impacting the initial bed surface and the particle velocity:

$$
J=\frac{Q}{(2 e h \tan \alpha) u}
$$

where $u=\sqrt{\left(\Delta \rho / \rho_{f}\right) g d}$ is the inertial particle velocity corrected from buoyancy effects with $g$ the gravitational acceleration and $\alpha$ the half-angle of the liquid jet. Note that $J$ corresponds to the square root of a Shields number based on the average velocity of the jet impacting the initial bed surface. The jet half-angle $\alpha$ is measured experimentally for each $(h, Q)$ in the absence of a granular layer, $h$ corresponding here to the distance between the injection nozzle and the horizontal wall at the cell bottom (see Supplemental Material A.4). As already proposed in the literature [15], the critical value $J \simeq 0.6$ captures well the transition between weak or no erosion, and the formation of a crater (Fig. 2, solid black line). In the following, we will consider the average angle evolution as a function of the flow-rate, $\alpha=\alpha_{0}+\left(\alpha^{*}-\alpha_{0}\right) Q / Q_{c}$ for $Q \leq Q_{c}$ and $\alpha=\alpha^{*}$ for $Q \geq Q_{c}$, with $\alpha_{0}=3^{\circ}, \alpha^{*}=6.5^{\circ}$ and $Q_{c}=2 \mathrm{~mL} / \mathrm{s}$ (Fig. S4, dashed line).

The exploration of different system states is quantified by introducing an asymmetry parameter, $\xi$, defined for each system state as $\left\langle Q_{1}\right\rangle /\left\langle Q_{1}+Q_{2}\right\rangle$ where $\langle$.$\rangle indicates$ the time average of the signal. We exclude here the transition regions. For $\xi=0.5,\left\langle Q_{1}\right\rangle=\left\langle Q_{2}\right\rangle$, while for $\xi=0$ or 1 , all the upward flow is locked on one side of the crater (fully asymmetric jet). All experiments corresponding to a same value of the dimensionless parameter $J$ are concatenated, so we can estimate not only the different system states, but also the probability $p$ of the system to explore such state in time. In addition, $\xi$ is divided in bins of width 0.1 , meaning that two states in the same bin are attributed the same $\xi$ value. Figure 3e summarizes the system states and liquid jet dynamics when increasing $J$. The markers size and color indicate the probability of the system to be in the state of value $\xi$, while the symbols indicate the crater geometry (open or close). Open craters (Fig. 3e, $\nabla$ ) always display a stationary behavior, symmetric fluxes and $\xi=0.5$. Close craters (Fig. 3e, $\bigcirc$ ), on the contrary, display non-stationary fluxes and explore different states in time. The transition from a stationary to a non-stationary jet occurs through a bifurcation diagram, with a transition at $J^{*} \simeq 1$. In the non-stationary regime, the system has an almost equal probability to explore the different states. The lack of data points for some $J$ (for instance $J \simeq 4$ ) is the consequence of the lack of statistics. Although binning $\xi$ makes possible in most cases to increase the statistics, it would require more or longer experiments to explore the whole range of $\xi$ expected from this figure.

When the jet is fully asymmetric $(\xi=0$ or 1$)$, it does not display oscillations. Oscillations develop gradually when $|\xi-1 / 2|$ decreases to zero (Fig. 3e, black arrows), until steady, quasi-periodic oscillations appear for $\xi=0.5$ 
(Fig. 3d). The oscillation frequency is quantified by performing the cross-correlation of the flow-rate fluctuations $\left(Q_{1}-\left\langle Q_{1}\right\rangle\right)$ with $\left(Q_{2}-\left\langle Q_{2}\right\rangle\right)$. Figure $4 \mathrm{a}$, inset, displays the oscillations frequency as a function of $J$, in the region where they develop $\left(J>J^{*}\right.$, gray region). The gray crosses indicate the frequency for each experiment, and the black star their average for the same $J$. To scale the frequency, we introduce the global Strouhal number $S t=f L / V$ based on the jet velocity at the injector outlet, $V=Q / S$, and on the impingement length $L=h$ [24]. St exhibits a power-law behavior as a function of $J, S t=\beta J^{\gamma}$ with $\gamma \simeq-1$ and $\beta \simeq 0.09$ (Figure 4a). Rewriting this law using Eq. 1 provides the frequency evolution as a function of the system's parameters:

$$
f=2 \beta\left(\frac{u}{S / e}\right) \tan \alpha .
$$

The oscillation frequency is therefore driven by the particle inertial velocity $u$, including buoyancy correction, the jet dispersion $\alpha$ and the ratio between the injector cross-section $S$ and the confinement length $e$. It only depends indirectly on the jet speed at the outlet through the jet half-angle $\alpha$. The dashed line in Figure 4, inset, corresponds to the frequency computed from equation 2 with $\alpha=\alpha^{*}$ (see Supplemental Material A.4), which is the case for most experiments showing oscillations $\left(Q \geq Q_{c}\right)$. It captures successfully the order of magnitude $(\simeq 2.7 \mathrm{~Hz})$ of the observed frequency, using the empirical parameter $\beta \simeq 0.09$.

The appearance of self-sustained oscillations for confined jets impinging an obstacle is commonly explained as a feedback mechanism resulting in the formation of a large, coherent structure $[24,25,28,30,31]$. Two possible mechanisms, acoustic or hydrodynamic, can be at the origin of the feedback loop: (1) the impinging jet generates acoustic waves which propagate upstream and interact with the nozzle; (2) downstream-convected structures are advected by a recirculation flow and force in-phase oscillations at the nozzle exit. These mechanisms, however, are not at stake here for the following reasons. (1) The acoustic feedback loop is characterized, at low Mach number, by a constant Strouhal number [30], which discards this origin for the observed oscillations. (2) Feedback effect due to the growth and recirculation of flow disturbances generates oscillations whose frequency increases with the jet speed [27], which is not observed in our experiments.

Figure $4 \mathrm{~b}$ presents the spatiotemporal evolution of the images intensity along a horizontal section of about $1 \mathrm{~cm}$ height encompassing the crater aperture. Each column is the average over the section height, and $x=0$ is at the vertical of the nozzle. The crater aperture width, $W$, exhibits clear oscillations, here with a frequency of about $2.5 \mathrm{~Hz}$ (white dashed lines), similar to the jet flow oscillations $(2.44 \mathrm{~Hz}$ for this experiment). We report in Figure $4 \mathrm{~b}$ the frequency inferred from the spatiotemporal
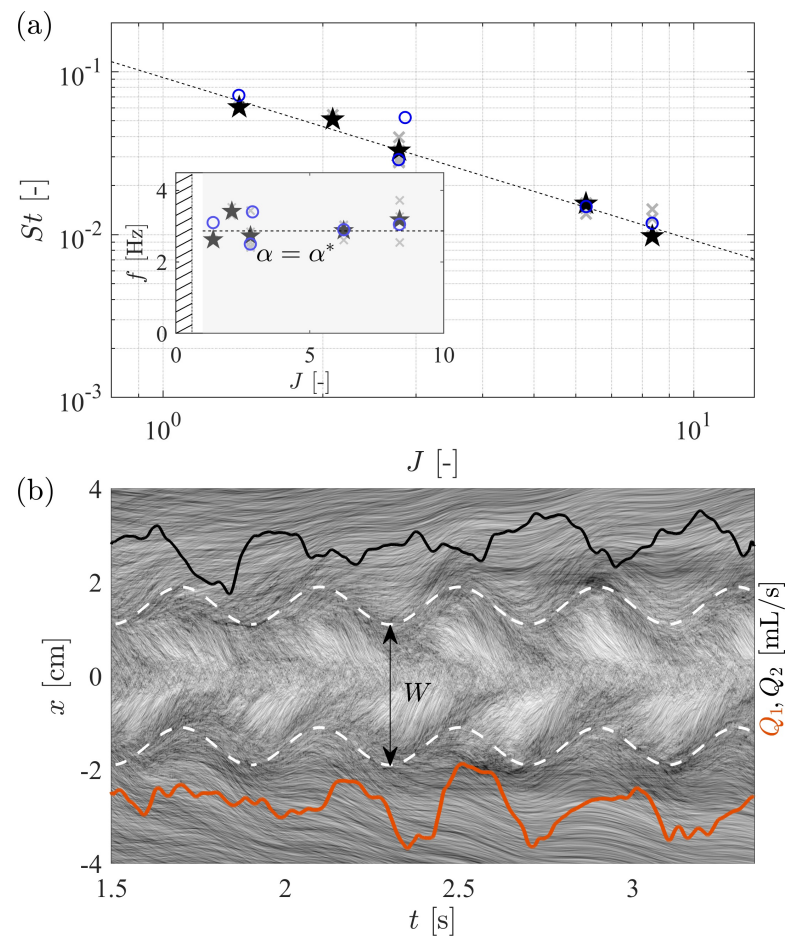

Figure 4. (a) Strouhal number $S t$ as a function of $J$ ( $\times$ from fluxes cross-correlation, $\star$ data from fluxes averaged for a given $J$, $\bigcirc$ from spatiotemporal). The errorbar is smaller than each symbol size. Dashed line: fit of the average values, $S t=\beta J^{-1}$ with $\beta \simeq 0.09$. Inset: jet oscillation frequency $f$. Hatched region: no or weak erosion; gray area: oscillations. Dashed line: frequency given by equation 2 for $\alpha=\alpha^{*}$. (b) Spatiotemporal evolution of the images intensity along a horizontal region encompassing the crater aperture. The crater aperture width $W$ is visible as the clear, oscillating region $[h=3 \mathrm{~cm}, Q=4 \mathrm{~mL} / \mathrm{s}]$. Orange \& black lines: data $Q_{1} \&$ $Q_{2}$ from Fig. 3d, vertically shifted for clarity. White dashed lines: harmonic oscillations at $2.5 \mathrm{~Hz}$ (guides for the eye).

diagram (blue circles) for different $J$. It matches quite fairly the frequency obtained from the flux signals analysis. These oscillations thus correspond to the quasiperiodic motion of grains forming the dunes at the crater aperture, visible in Fig. 1d. The self-sustained oscillations are therefore generated by a coupling between the fluid and grains, which explains why the frequency is almost independent on the jet speed $\left(\alpha=\alpha^{*}\right.$ in most experiments with oscillations) but strongly depends on the inertial particle velocity $u$.

Impinging jets over deformable granular beds in confined environment thus lead to the appearance of flow oscillations for close crater geometries. Although further dependence with the confinement should be assessed, this study shows that the strong coupling between fluid and particles is responsible for this instabilities generation, which could be responsible for safety failure in many practical situations.

This work was supported by Programa de Coop- 
eración Científica ECOS/CONICYT C14E07, the Laboratoire International Associé "Matière : Structure et Dynamique" (LIA-MSD, France-Chile), the French research program ANR-16-CE30-0028 and IDEXLYON of the University of Lyon in the framework of the French program "Programme Investissements d'Avenir" (ANR16-IDEX-0005). The authors wish to thank the two anonymous referees for fruitful discussions which greatly improved this manuscript.

[1] A.M. Walsh, K.E. Holloway, P. Habdas, J.R. de Bruyn, Physical Review Letters 91(10), 104301 (2003)

[2] D. van der Meer, Annual Review of Fluid Mechanics 49, 463 (2017)

[3] L. Roberts, in A compilation of recent research related to the Apollo Mission (National Aeronautics and Space Administration, Hampton, Va., 1963), pp. 155-170

[4] P.T. Metzger, C.D. Immer, C.M. Donahue, B.T. Vu, R.C. Latta, M. Deyo-Svendsen, Journal of Aerospace Engineering 22(1), 24 (2009)

[5] K.J. Berger, A. Anand, P.T. Metzger, C.M. Hrenya, Physical Review E 87, 022205 (2013)

[6] A.H. Clark, R.P. Behringer, Granular Matter 16(4), 433 (2014)

[7] J.E. Lane, P.T. Metzger, Acta Geophysica 63(2), 568 (2015)

[8] H.U. Oebius, H.J. Becker, S. Rolinski, J.A. Jankowski, Deep-Sea Research II 48, 3453 (2001)

[9] Schriever, G. and Thiel, H., in Proceedings of the Tenth (2013) ISOPE Ocean Mining and Gas Hydrates Symposium (The International Society of Offshore and Polar Engineers (ISOPE), Szczecin, Poland, September 22-26, 2013, 2013), pp. 5-17

[10] S. Tachibana, M. Abe, M. Arakawa, M. Fujimoto, Y. Iijima, M. Ishiguro, K. Kitazato, N. Kobayashi, N. Namiki, T. Okada, R. Okazaki, H. Sawada, S. Sugita, Y. Takano, S. Tanaka, S. Watanabe, M. Yoshikawa, H. Kuninaka, The Hayabusa2 Project Team, Geochemical Journal 48, 571 (2014)

[11] T. Saiki, H. Imamura, M. Arakawa, K. Wada, Y. Takagi, M. Hayakawa, K. Shirai, H. Yano, C. Okamoto, Space Sci. Rev. 208, 165 (2017)

[12] E.B. Bierhaus, B.C. Clark, J.W. Harris, K.S. Payne, R.D.
Dubisher, D.W. Wurts, R.A. Hund, R.M. Kuhns, T.M. Linn, J.L. Wood, A.J. May, J.P. Dworkin, E. Beshore, D.S. Lauretta, the OSIRIS-REx Team, Space Sci. Rev. 214, 107 (2018)

[13] H.J. Becker, B. Grupe, H.U. Oebius, F. Liu, Deep-Sea Research II 48, 3609 (2001)

[14] T. Peacock, M.H. Alford, Scientific American 318(5), 72 (2018)

[15] O. Aderibigbe, N. Rajaratnam, Journal of Hydraulic Research 34(1), 19 (1996)

[16] P.T. Metzger, R.C. Latta, J.M. Schuler, C.D. Immer, AIP Conference Proceedings 1145(1), 767 (2009)

[17] P. Aussillous, J. Chauchat, M. Pailha, M. Médale, E. Guazzelli, Journal of Fluid Mechanics 736, 594 (2013)

[18] S. Badr, G. Gauthier, P. Gondret, Physics of Fluids 26(2), 023302 (2014)

[19] B.R. Sutherland, S. Dalziel, Physics of Fluids 26(3), 035103 (2014)

[20] C.Q. Lamarche, J. Sinclair Curtis, Chemical Engineering Science 138, 432 (2015)

[21] S. Badr, G. Gauthier, P. Gondret, Physics of Fluids 28(3), 033305 (2016)

[22] M. Houssais, C.P. Ortiz, D.J. Durian, D.J. Jerolmack, Phys. Rev. E 94, 062609 (2016)

[23] C. Sondhaus, Ann. Phys. 91(128) (1854)

[24] D. Rockwell, E. Naudascher, Annual Review of Fluid Mechanics 11(1), 67 (1979)

[25] C.M. Ho, N.S. Nosseir, Journal of Fluid Mechanics 105, 119 (1981)

[26] D. Rockwell, AIAA Journal 21(5), 645 (1983)

[27] E. Villermaux, E. Hopfinger, Physica D: Nonlinear Phenomena 72(3), 230 (1994)

[28] A. Maurel, P. Ern, B.J.A. Zielinska, J.E. Wesfreid, Phys. Rev. E 54(4), 3643 (1996)

[29] B.M. Gebert, M.R. Davidson, M.J. Rudman, Appl. Math. Model. 22, 843 (1998)

[30] D. Varieras, P. Brancher, A. Giovannini, Flow Turbul. Combust. 78, 1 (2007)

[31] B.W. Righolt, S. Kenjereš, R. Kalter, M.J. Tummers, C.R. Kleijn, Phys. Fluids 27, 095107 (2015)

[32] Y. Li, X. Ming, European Journal of Mechanics B 57, 40 (2016)

[33] W. Thielicke, E.J. Stamhuis, Journal of Open Research Software 2(1), e30 (2014)

[34] W. Thielicke, E.J. Stamhuis. PIVlab - Time-Resolved Digital Particle Image Velocimetry Tool for MATLAB (version: 2.02) (2014) 